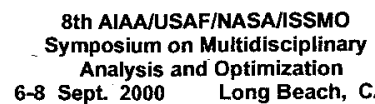

A00-40035

AIAA-2000-4751

\title{
ACCURACY CONSIDERATIONS IN APPROXIMATE REANALYSIS OF STRUCTURES
}

\author{
U. Kirsch ${ }^{*}$ and P.Y. Papalambrost \\ University of Michigan, Ann Arbor, MI 48109, USA
}

\begin{abstract}
Combined Approximations (CA) is an efficient method for reanalysis of structures where binomial series terms are used as basis vectors in reduced basis approximations. In previous studies high quality approximations have been achieved for large changes in the design, but the reasons for the high accuracy were not fully understood. In this paper some typical cases, where exact and accurate solutions are achieved by the method are presented and discussed. Exact solutions are obtained in the general case where a basis vector is a linear combination of the previous vectors. Such solutions are obtained also in cases of low rank modifications to structures or scaling of the initial stiffness matrix. In general the CA method does not provide exact solutions, but the solutions presented in the paper explain the high accuracy achieved with only small number of basis vectors. Accurate solutions are achieved in many cases where the basis vectors come close to being linearly dependent. Such solutions are achieved also in cases of changes in a small number of elements or when the angle between the two vectors representing the initial design and modified design is small. Numerical examples of various changes in cross sections of elements and in the layout of the structure show that accurate results are achieved even in cases where the series of basis vectors diverges.
\end{abstract}

\section{INTRODUCTION}

Multiple repeated analyses are needed in various design and optimization problems. In general, the structural response cannot be expressed explicitly in terms of the structure properties, and structural analysis involves the solution of a set of simultaneous equations. Reanalysis methods are intended to analyze efficiently structures modified due to changes in the design.

Approximate reanalysis methods have been used extensively in structural optimization to reduce the number of exact analyses and the overall computational cost during the solution process. The Combined Approximations (CA) method developed recently is considered in this paper. The method combines several concepts and methods such as reduced basis, series approximations, matrix factorization and Gram-Schmidt orthonormalization.

\footnotetext{
* Visiting Professor, Dept. of Mechanical Engineering.

$\dagger$ Professor, Dept. of Mechanical Engineering.

Copyright c 2000 by U. Kirsch and P.Y. Papalambros.

Published by the American Institute of Acronautics and Astronautics Inc. with permission.
}

The effectiveness of the method in various optimization problems has been demonstrated in previous studies $^{1-5}$. Initially the CA method was used only for linear reanalysis models. Recently, the method has been used successfully also in eigenvalue $^{6}$ and nonlinear analysis ${ }^{7}$ problems. Applications of the method in a large variety of problems is discussed elsewhere ${ }^{8}$.

High quality approximations of the structural response for large changes in the design have been achieved in previous studies, but the reasons for the high accuracy were not fully understood. In this paper some typical cases, where exact and accurate solutions are achieved by the CA method, are presented and discussed. In general the CA method does not provide exact solutions, but the solutions presented in the paper explain the high accuracy achieved with only a small number of basis vectors. The solution procedure is briefly described in Sect. 2 . Three typical cases, where exact solutions are achieved by the CA method, are introduced and discussed in Sect. 3. Exact solutions are obtained in the general case where a basis vector is a linear combination of the previous vectors. Such solutions are obtained also in cases of low rank modifications to structures or scaling of the initial stiffness matrix. Various cases of accurate solutions are discussed in Sect. 4. Convergence properties of the series of basis vectors and the series of the CA terms are presented, and criteria intended to evaluate the errors in the approximations are introduced. Accurate solutions are achieved in many cases where the basis vectors come close to being linearly dependent. Such solutions are achieved also in cases of changes in small number of elements or when the angle between the two vectors representing the initial and modified design is small. Numerical examples illustrating the accuracy of the results are included in Sect. 5.

\section{APPROXIMATE REANALYSIS}

Consider an initial design with stiffness matrix $\mathbf{K}^{*}$ and corresponding displacements $\mathbf{r}^{*}$ computed by the stiffness analysis equations

$$
\mathbf{K}^{*} \mathbf{r}^{*}=\mathbf{R}
$$

For simplicity of presentation the load vector $\mathbf{R}$ is assumed to be constant, but the procedure presented is suitable also when the elements of $\mathbf{R}$ are functions of the design variables. Assume a change in the design so that the modified stiffness matrix is given by

$$
\mathbf{K}=\mathbf{K}^{*}+\Delta \mathbf{K}
$$


where $\Delta \mathbf{K}$ is the corresponding change in the stiffness matrix. The object is to evaluate the modified displacements $\mathbf{r}$ due to various changes $\Delta \mathbf{K}$ efficiently and accurately, without solving the complete set of modified implicit equations

$$
\mathbf{K} \mathbf{r}=\left(\mathbf{K}^{*}+\Delta \mathbf{K}\right) \mathbf{r}=\mathbf{R}
$$

Evaluation of the modified displacements by the CA method is briefly described subsequently.

We assume that the displacement vector of a modified design can be approximated by a linear combination of $s$ linearly independent basis vectors as

$$
\mathbf{r}=\mathrm{y}_{1} \mathbf{r}_{1}+\mathrm{y}_{2} \mathbf{r}_{2}+\ldots+\mathrm{y}_{s} \mathbf{r}_{s}=\mathbf{r}_{B} \mathbf{y}
$$

where $\mathbf{r}_{B}$ is the matrix of $s$ basis vectors and $\mathbf{y}$ is a vector of the $s$ coefficients to be determined. Defining matrix $\mathbf{B}$ by

$$
\mathbf{B}=\mathbf{K}^{*-1} \Delta \mathbf{K}
$$

the basis vectors are then given by the terms of the binomial series

$$
\mathbf{r}_{1}=\mathbf{r}^{*} \mathbf{r}_{2}=-\mathbf{B} \mathbf{r}^{*} \quad \mathbf{r}_{\mathrm{s}}=-\mathbf{B}^{\mathrm{s}-1} \mathbf{r}^{*}
$$

Calculation of the series terms involves only forward and backward substitutions in cases where the initial stiffness matrix $\mathbf{K}^{*}$ is given in a decomposed form from the initial analysis 5 .

To determine the vector of coefficients $\mathbf{y}$, the approximate displacements of Eq. (4) are substituted into the modified analysis Eqs. (3). Premultiplying the resulting equation by $\mathbf{r}_{B}^{T}$ yields

$$
\mathbf{K}_{R} \mathbf{y}=\mathbf{R}_{R}
$$

where

$$
\mathbf{K}_{R}=\mathbf{r}_{B}^{T} \mathbf{K} \mathbf{r}_{B} \quad \mathbf{R}_{R}=\mathbf{r}_{B}^{T} \mathbf{R}
$$

For cases where $s$ is much smaller than the number of degrees of freedom $n$, the approximate displacement vector can be evaluated by solving the smaller $(s \mathrm{x} s)$ system in Eq. (7) for $y$ instead of computing the exact solution by solving the large ( $n \times n)$ system in Eq. (3). The final displacements are then computed for the given $\mathbf{y}$ by Eq. (4).

In summary, evaluation of the modified displacements by the CA method involves the following steps:

a. The modified stiffness matrix $\mathbf{K}$ is first introduced [Eq. (3)]. Since $\mathbf{K}^{*}$ is already given, this step involves only calculation of $\Delta \mathbf{K}$.

b. The basis vectors $\mathbf{r}_{i}$ are calculated by Eq. (6). Calculation of each basis vector involves only forward and backward substitutions. c. The reduced matrix $\mathbf{K}_{R}$ and the reduced vector $\mathbf{R}_{R}$ are calculated by Eq. (8).

d. The unknown coefficients $\mathbf{y}$ are calculated by solving the set of ( $s \times s$ ) equations (7).

e. The displacements $\mathbf{r}$ are evaluated by Eq. (4).

The solution process is based on results of a single exact analysis, and it is suitable for different types of structures and design changes. The method is easy to implement, it can be used readily with a general finite element program, and calculation of derivatives is not required.

The efficiency of reanalysis by the CA method, compared with complete analysis of the modified design, can be measured by various criteria, e.g. the CPU effort or the number of algebraic operations. It is then possible to relate the computational effort to various parameters such as the number of degrees of freedom, the number of basis vectors considered and the accuracy of the results. It has been found that calculation of each basis vector involves about $2 \%$ of the CPU time needed for complete analysis. In many cases a small number of basis vectors is sufficient to achieve adequate accuracy. For moderate changes in the design 2-3 vectors are often sufficient whereas 5 6 vectors might be needed for large changes. Considering the latter number of vectors results for various problems showed that the total CPU effort, compared with complete analysis of the modified design, has been reduced by more than $75 \%$.

\section{EXACT SOLUTIONS}

In this section the following three typical cases, where exact solutions are obtained by the CA method, are presented:

a. A general case, where a basis vector is a linear combination of the previous vectors, is developed in Sect. 3.1. In many cases, where the basis vectors come close to being linearly dependent, accurate solutions are achieved by the CA method.

b. The case of low rank modifications to structures, where the number of modified elements in the stiffness matrix is limited, is presented in Sect. 3.2. The exact solution is achieved if one basis vector is introduced for each changed member. If some of the vectors are linearly dependent, the exact solution is achieved with a smaller number of vectors.

c. The common case of scaling of the initial stiffness matrix, where exact solutions are obtained by consideration of a single basis vector, is presented in Sect. 3.3. In many cases, where the angle between the two vectors representing the initial design and modified design is small, accurate solutions are achieved by the CA method. 


\subsection{Linearly dependent basis vectors}

A general case of changes in the structure, where exact solution is obtained by the CA method, is presented subsequently. To obtain a convenient expression for the exact solution of the modified design, premultiply Eq. (3) by $\mathbf{K}^{*-1}$ and substitute Eqs. (1) and (5). The result is

$$
(\mathbf{I}+\mathbf{B}) \mathbf{r}=\mathbf{r}^{*}
$$

Premultipling Eq. (9) by $(\mathbf{I}+\mathbf{B})^{-1}$ gives the exact modified displacements

$$
\mathbf{r}=(\mathbf{I}+\mathbf{B})^{-1} \mathbf{r}^{*}
$$

To obtain a convenient expression for the approximate solution in terms of assumed $s$ basis vectors, substitute the expressions of the basis vectors [Eq. (6)] into Eq. (4). The resulting CA expression is

$$
\mathbf{r}=\mathrm{y}_{1} \mathbf{r}^{*}-\mathrm{y}_{2} \mathbf{B} \mathbf{r}^{*}+\mathrm{y}_{3} \mathbf{B}^{2} \mathbf{r}^{*}-\ldots+\mathrm{y}_{s} \mathbf{B}^{\mathrm{s}-1} \mathbf{r}^{*}
$$

Assuming that the approximate expression of Eq (11) is equal to the exact solution of Eq. (10), premultiplying both equations by $(\mathbf{I}+\mathbf{B})$ and rearranging gives the linear expression

$$
\mathbf{r}_{s+1}=\sum_{i=1}^{s} a_{i} \mathbf{r}_{i}
$$

where $a_{i}$ are scalar multipliers given by

$$
\begin{gathered}
a_{1}=\left(y_{1}-1\right) / y_{s} \\
a_{i}=\left(y_{i}-y_{i-1}\right) / y_{s} \quad i=2,3, \ldots, s
\end{gathered}
$$

Equation (12) shows that when the reduced basis expression with $s$ terms is equal to the exact solution, then the $s+1$ basis vector is a linear combination of the previous $s$ vectors. That is, the $s+1$ basis vectors are linearly dependent.

\subsection{Simultaneous rank one changes}

Exact methods are efficient in cases of low rank modifications to structures and are applicable to situations where the number of modified elements in the stiffness matrix is limited. These methods are usually based on the Sherman-Morrison ${ }^{9}$ and Woodbury ${ }^{10}$ formulae for the update of the inverse of a matrix. It has been shown recently ${ }^{11}$ that various reanalysis methods may be viewed as variants of these formulae. In particular, exact solutions obtained by the CA method and Sherman-MorrisonWoodbury formulae are equivalent.

Consider for example the case of simultaneous changes in $m$ truss members. The exact solution is obtained if one basis vector is selected for each changed member ${ }^{3}$

$$
\mathbf{r}_{i}=\mathbf{K}_{0}^{-1} \Delta \mathbf{K}_{i} \mathbf{r}_{0} \quad i=1, \ldots, m
$$

where $\Delta \mathbf{K}_{i}$ is the contribution of the $i$ th member to $\Delta \mathbf{K}$. The exact solution is given by

$$
\mathbf{r}=\mathbf{r}_{0}+\sum_{i=1}^{m} y_{i} \mathbf{r}_{i}
$$

where $\mathbf{r}_{0}$ is the vector of initial displacements. This procedure is efficient when the number of changed members is much smaller than the number of degrees of freedom. Exact solutions achieved by the CA method and the Sherman-Morrison-Woodbury formulae in such cases are equivalent. If some of the vectors are linearly dependent, the exact solution is obtained with a smaller number of vectors.

\subsection{Scaling of the initial stiffness matrix}

Scaling of the initial stiffness matrix $\mathbf{K}^{*}$ is carried out by multiplying the latter matrix by a positive scaling multiplier $\mu$ to obtain the modified matrix

$$
\mathbf{K}=\mu \mathbf{K}^{*}
$$

From Eqs. (1), (3) and (16) it is clear that the exact displacements after scaling can be calculated directly by

$$
\mathbf{r}=\mu^{-1} \mathbf{r}^{*}
$$

The condition of Eq. (16) requires linear dependence of the stiffness matrix on the change in the design. In general, the elements of $\mathbf{K}$ are some nonlinear functions of the design variables. A typical case where the condition of Eq. (16) is satisfied is scaling of the cross sections or the geometry of a truss structure, where the lengths of all elements are multiplied by $\mu$ and their direction is unchanged.

Consider the case where the modified design is a scaled design $\mu \mathbf{K}^{*}$, as given by Eq. (16). Then, from Eq. (5)

$$
\mathbf{B}=\mathbf{K}^{*-1} \Delta \mathbf{K}=(\mu-1) \mathbf{I}
$$

where $\mathbf{I}$ is an identity matrix. The resulting basis vectors [Eqs. (6)] become linearly dependent

$\mathbf{r}_{1}=\mathbf{r}^{*} \quad \mathbf{r}_{2}=-(\mu-1) \mathbf{r}^{*} \quad \mathbf{r}_{3}=(\mu-1)^{2} \mathbf{r}^{*}$

Thus, the exact modified displacements are determined directly by the first basis vector, and no approximations are needed. Consideration of a single basis vector with a coefficient $y_{1}=\mu^{-1}$ will provide the exact solution as given by Eq. (17).

\section{ACCURACY CONSIDERATIONS}

\subsection{Convergence of the series}

In this section convergence considerations related to the series of basis vectors and the series of the CA terms are presented. 
The series of basis vectors converges if and only if

$$
\lim _{k \rightarrow \infty} \mathbf{B}^{k}=\mathbf{0}
$$

A sufficient criterion for the convergence of the series is that $\|\mathbf{B}\| \leq 1$, where $\|\mathbf{B}\|$ is the norm of $\mathbf{B}$. It can be shown that $\rho(\mathbf{B}) \leq\|\mathbf{B}\|$, where $\rho(\mathbf{B})$ is the spectral radius, i.e. the largest eigenvalue of matrix B. From the above, a sufficient condition for convergence is $\rho(\mathbf{B}) \leq 1$.

It is convenient to express the change in the design $\Delta \mathbf{K}[\mathrm{Eq} .(2)]$ as

$$
\Delta \mathbf{K}=\alpha \Delta \mathbf{K}^{*}
$$

Where $\Delta \mathbf{K}^{*}$ is a matrix representing the direction of change and $\alpha$ is a scalar multiplier describing the magnitude of change in the design. In the solution process, the basis vectors $\mathbf{r}_{i}$ are determined by Eq. (6) and are multiplied by the corresponding scalars $y_{i}$ to obtain the final displacements [Eq. (4)]. Multiplying a basis vector $\mathbf{r}_{i}$ by any scalar will not change the approximate solution (only the corresponding scalar $y_{i}$ will be changed). Therefore, identical basis vectors can be selected for any given $\Delta \mathbf{K}^{*}$ and different $\alpha$ values. In cases where the elements of the basis vectors become very large due to large $\Delta \mathbf{K}$ values, it is possible to normalize a basis vector $\mathbf{r}_{i}$ by dividing it by an arbitrary reference element of the vector (say, the first element $r_{1 i}$ ) to obtain

$$
\mathbf{r}_{N i}=\mathbf{r}_{i} / \mathbf{r}_{1 i}
$$

This operation scales the first element of the vector to unity and, as noted earlier, it does not change the approximate solution.

To evaluate the accuracy of the approximations, an uncoupled set of new basis vectors $\mathbf{V}_{i}(i=1, \ldots, s)$ is introduced using a Gram-Schmidt orthogonalization and normalization method ${ }^{5,7}$. The new vectors are determined by the original ones $\mathbf{r}_{i}$ from

$$
\begin{gathered}
\mathbf{V}_{1}=\left|\mathbf{r}_{1}^{T} \mathbf{K} \mathbf{r}_{1}\right|^{-1 / 2} \mathbf{r}_{1} \\
\overline{\mathbf{V}}_{i}=\mathbf{r}_{i}-\sum_{j=1}^{i-1}\left(\mathbf{r}_{i}^{T} \mathbf{K} \mathbf{V}_{j}\right) \mathbf{V}_{j} \\
\mathbf{V}_{i}=\left|\overline{\mathbf{V}}_{i}^{T} \mathbf{K} \overline{\mathbf{V}}_{i}\right|^{-1 / 2} \overline{\mathbf{V}}_{i} \quad i=2, \ldots, s
\end{gathered}
$$

where $\overline{\mathbf{V}}_{i}$ and $\mathbf{V}_{i}$ are the $i$ th non-normalized and normalized vectors, respectively. Defining the matrix $\mathbf{V}_{B}$ of new basis vectors and the vector $\mathbf{z}$ of new coefficients, the reduced system of Eq. (7) becomes uncoupled and the final displacements are given by the explicit expression

$$
\mathbf{r}=\mathbf{V}_{B} \quad \mathbf{z}=\mathbf{V}_{B}\left(\mathbf{V}_{B}^{T} \mathbf{R}\right)
$$

Equation (25) can be expressed as an additively separable quadratic function of the vectors $\mathbf{V}_{i}$ by

$$
\mathbf{r}=\sum_{i=1}^{s} \mathbf{V}_{i}\left(\mathbf{V}_{i}^{T} \mathbf{R}\right)
$$

One advantage in using the new vectors is that all expressions for evaluating the displacements are explicit functions of the original basis vectors. Calculation of any new basis vector $\mathbf{V}_{i}$ results in an additional term of the displacements expression [Eq. (26)] that is a function of the original vectors $\mathbf{r}_{j}(j=1$, $2, \ldots, i)$. Consequently, additional vectors can be considered without modifying the calculations that were carried out already.

For any assumed number of basis vectors, the results obtained by considering either the original set of basis vectors or the new set of uncoupled basis vectors are identical. While the normalized vectors $\mathbf{V}_{i}$ are of similar magnitude, the values of the $z_{i}$ coefficients and the corresponding terms of the series of Eq. (26) are gradually decreased. It will be shown later that transforming the binomial series terms [Eq. (6)] into the terms of the CA series [Eq. (26)] provides accurate solutions even in cases where the binomial series diverges.

The accuracy of the results for a specific number $s$ of basis vectors can be evaluated by the $s$ th term, $\mathbf{r}^{(\mathrm{s})}$, of the approximate displacements expression [Eq. (26)], given by

$$
\mathbf{r}^{(s)}=\mathbf{V}_{s}\left(\mathbf{V}_{s}^{T} \mathbf{R}\right)
$$

If the solution process converges, the size of the elements of the vector $\mathbf{r}^{(\mathrm{s})}$, in Eq. (27) can be used as a convergence criterion.

\subsection{Accurate solutions}

It has been noted that in general the CA method does not provide exact solutions, but in many cases accurate solutions are achieved with only a small number of basis vectors. The various cases of exact solutions presented in Sect. 3 explain the high accuracy achieved by the method. In this section some cases where accurate solutions are expected will be presented.

Nearly linear dependent basis vectors. It has been shown in Sect. 3.1 that exact solutions are obtained in cases where the basis vectors are linearly dependent. Consequently, it is expected that accurate solutions would be achieved in cases were the basis vectors come close to being linearly dependent. Two basis vectors $\mathbf{r}_{i}$ and $\mathbf{r}_{i+1}$ are close to being linearly dependent if

$$
\cos \beta_{i+1}=\left(\mathbf{r}_{i}^{T} \mathbf{B}^{*} \mathbf{r}_{i}\right) /\left(\left|\mathbf{r}_{i}\right|\left|\mathbf{B}^{*} \mathbf{r}_{i}\right|\right) \cdot 1
$$


where $\beta_{i+1}$ is the angle between the two vectors and II denotes the absolute value. It can noted [Eq. (21)] that for any given $\Delta \mathbf{K}^{*}$, the angle $\beta_{i} i+1$ is independent of the scalar $\alpha$. It will be shown by numerical examples that the basis vectors determined by the CA method satisfy the condition of Eq. (28), as $i$ is increased, even in cases of very large changes in the design.

Changes in limited number of elements. It has been noted in Sect. 3.2 that in the case of simultaneous $m$ rank-one changes, exact solutions are obtained by the CA method if one basis vector is selected for each changed member. If some of the basis vectors are linearly dependent, the exact solution is achieved for a smaller number of vectors. It has been observed that in many cases where limited number of elements are changed, exact solutions are achieved by the CA method with small number of basis vectors. Results will be demonstrated by numerical examples in Sect. 5 .

Nearly scaled designs. Consider the case where the change in the stiffness matrix $\Delta \mathbf{K}$ [Eq. (21)] can be expressed in terms of corresponding change in the design variables $\mathbf{X}$ by

$$
\mathbf{X}=\mathbf{X}^{*}+\Delta \mathbf{X}=\mathbf{X}^{*}+\alpha \Delta \mathbf{X}^{*}
$$

Both the direction of change $\Delta \mathbf{X}^{*}$ and the magnitude of change $\alpha$ may affect significantly the accuracy of the approximations. The effect of both can be represented by the single parameter $\theta$, namely the angle between the vector of the modified design $\mathbf{X}$ and the vector of initial design $\mathbf{X}^{*}$, given by

$$
\cos \theta=\left(\mathbf{X}^{T} \mathbf{X}^{*}\right) /\left(|\mathbf{X}| \mid \mathbf{X}^{*}\right)
$$

Various designs, obtained by scaling a certain modified design, provide identical $\theta$ angles. For example, Fig. 1 illustrates how the two modified designs $A$ at $\mathbf{X}=\mathbf{X}^{*}+0.1 \Delta \mathbf{X}^{*}$, and $D$ at $\mathbf{X}=10\left(\mathbf{X}^{*}+0.1 \Delta \mathbf{X}^{*}\right)$, correspond to an identical $\theta$. It will be shown in Sect. 5 that high accuracy is achieved with a small number of basis vectors for designs $A$ (representing a small change in the design) and $D$ (representing a very large change in the design), which both correspond to a small $\theta$ value. More basis vectors are needed for designs $B$ and $C$ that correspond to larger $\theta$. It should be emphasized that in the present discussion only the space formed by the vectors $\mathbf{X}^{*}$ and $\mathbf{X}$ is considered. For the complete design space, smaller $\theta$ values not always guarantee better approximations.

For any given direction vector $\Delta \mathbf{X}^{*}$, the magnitude of change $\alpha$ determines the value of $\theta$ and the accuracy of the results. The larger $\alpha$ is, the larger is the angle $\theta$, and more basis vectors might be required to achieve adequate accuracy. Since accurate results are expected for small angles $\theta$, it might prove useful to apply the angle constraints ${ }^{13}$

$$
\theta^{L} \leq \theta \leq \theta^{U}
$$

where $\theta^{L}$ and $\theta^{U}$ are predetermined limits.

Common limitations on changes in the design, are the move limit constraints

$$
\Delta \mathbf{X}^{L} \leq \Delta \mathbf{X} \leq \Delta \mathbf{X}^{U}
$$

where $\Delta \mathbf{X}^{L}$ and $\Delta \mathbf{X}^{U}$ are predetermined lower and upper limits, respectively, on the design variable changes. An alternative approach, used in trust region algorithms ${ }^{12}$, is to restrict the solutions to some region around $\mathbf{X}^{*}$ by constraints of the form

$$
\|\Delta \mathbf{X}\| \cdot \Delta
$$

in which $\Delta$ is the radius of the region to be restricted. Constraints of the type of Eqs. (32) and (33) might be effective only for local approximations (such as the Taylor series), where small changes in the design variables are assumed. They are not suitable for the CA method where accurate solutions are obtained for large changes in the design.

\section{NUMERICAL EXAMPLES}

\subsection{Various design changes}

Consider the classic ten-bar truss problem shown in Fig. 2 with a single loading condition of two concentrated loads. The design variables are the members' cross-sectional areas, the initial cross sections equal unity, the modulus of elasticity is 30000 , and the analysis unknowns are the horizontal and vertical displacements at joints 1,2, 3 and 4, respectively. The stress constraints are $-25 \leq \sigma \leq 25$, and the minimum size constraints are $0.001 \leq \mathbf{X}$. Assuming the weight as an objective function, the resulting optimal design is

$$
\begin{array}{r}
\mathbf{X}_{\text {opt }}^{T}=\{8.0,0.001,8.0,4.0,0.001,0.001,5.667 \\
5.667,5.667,0.001\}
\end{array}
$$

The line from the initial design to the optimal design is given by

$$
\mathbf{X}=\mathbf{X}^{*}+\alpha \Delta \mathbf{X}^{*}
$$

where $\Delta \mathbf{X}^{*}$ is defined as

$$
\begin{array}{r}
\Delta \mathbf{X}^{* T}=\{7.0,-0.999,7.0,3.0,-0.999,-0.999 \\
4.667,4.667,4.667,-0.999\}
\end{array}
$$

To illustrate the effect of various design changes on the accuracy of the results, four typical cases were considered (see Fig. 1): 
A. Small change in the design (up to $-10 \%$ and $+70 \%)$ and small angle $\theta\left(\alpha=0.1, \theta=14^{\circ}\right)$. The modified design is given by $\mathbf{X}=\mathbf{X}^{*}+0.1 \Delta \mathbf{X}^{*}$.

$B$. Medium change in the design (up to $-50 \%$ and $+350 \%)$ and medium angle $\theta\left(\alpha=0.5, \theta=34^{\circ}\right)$. The modified design is given by $X=X^{*}+0.5 \Delta X^{*}$.

$C$. Large change in the design (up to $-100 \%$ and $+700 \%$ ) and large angle $\theta$ (the optimum, $\left.\alpha=1.0, \theta=41^{0}\right)$. The modified design is given by $\mathbf{X}=\mathbf{X}^{*}+\Delta \mathbf{X}^{*}$.

$D$. Very large change in the design (up to $+1600 \%)$ and small angle $\theta\left(\theta=14^{\circ}\right)$. The modified design is given by scaling design Case $B$ by a factor of $10, \mathbf{X}=10\left(\mathbf{X}^{*}+0.1 \Delta \mathbf{X}^{*}\right)$.

Results obtained for the above cases and various numbers of basis vectors by the CA method are summarized in Table 1. An accurate solution (maximum displacement error of 0.05 ) is achieved with only two basis vectors for cases $A$ and $D$. Similar accuracy is achieved in both cases with identical small $\theta$ values, although the design change in Case $D$ is much larger. The accuracy in Case $D$ is higher than that of Case $C$, although the design change in the latter case is smaller (but the angle $\theta$ is larger). For a given direction $\Delta \mathbf{X}^{*}$, the number of basis vectors needed to achieve a certain accuracy is increased with $\alpha$. Accurate solution (maximum displacement error of 0.05 ) is achieved with three basis vectors for Case $B \quad(\alpha=0.5)$ and with four basis vectors for case $C(\alpha=1)$.

Considering Case $C(\alpha=1)$, Fig. 3 shows how the norm of the basis vectors (the binomial series terms) is increased and the series diverges. Figure 4 shows the norm of the uncoupled basis vectors $V_{i}$, and Fig. 5 shows how the norm of the CA terms $V_{i} z_{i}$ is decreased and the series converges as the number of basis vectors is increased.

In summary, accurate results are obtained even in cases where the series of basis vectors (the binomial series) diverges. Finally, Table 2 shows that the basis vectors determined by the CA method are close to being linearly dependent even in cases of very large changes in the design. Identical $\beta$ values are obtained for design Cases $A, B$ and $C$, having identical direction of change $\Delta \mathbf{X}^{*}$.

\subsection{Change in small number of elements}

To illustrate numerical results for cases where the number of changed elements is small, consider again the initial ten-bar truss shown in Fig. 2. The following cases of changes in the topology by deletion of members and joints have been solved (see the modified topologies in Fig. 6):

a. Deletion of members $2+6+10$ and joint 2 .

b. Deletion of members $2+5+6+10$ and joint 2 .

c. Deletion of members $2+6+7+10$ and joint 2 .

d. Deletion of members $2+6+8+10$ and joint 2 .
The exact solutions, summarized in Table 3 , have been achieved for all the above cases with only three basis vectors.

\subsection{Fifty-bar truss, nearly scaled designs}

Consider the cantilever truss shown in Fig. 7a. The truss is subjected to a single load at the end and all cross section areas equals unity. The modulus of elasticity is 10000 and the forty unknowns are the horizontal (to the right) and the vertical (upward) displacements at joints 2 through 21 , respectively. Two geometric variables have been considered, the depth $D$ and the width $10 \mathrm{~W}$. Exact solution is achieved with a single basis vector for all designs where the ratio between the depth and the width of the truss does not change. The reason is that the vertical and the horizontal joint coordinates are changed simultaneously such that the geometry is scaled (the lengths of all members are changed by the same percentage whereas the direction cosines are unchanged). Assuming the initial geometry $D=$ $W=1.0$, two cases of geometrical changes have been solved :

a. The modified geometry is given by $D=1.2$ (a change of $20 \%$ in the depth, Fig. 7b).

b. The modified geometry is given by $D=2.0, \mathrm{~W}$ $=1.9$ (a change of $100 \%$ in the depth and $90 \%$ in the width, Fig. 7c).

The stiffness coefficients of thirty members have been changed, therefore exact reanalysis is not efficient. Assuming only two basis vectors (firstorder approximations, CA1), the results are given in Table 4. Comparing the results obtained for the two cases of geometrical modifications it can be seen that better approximations have been achieved in case $b$, for larger changes in the geometry. The better results in case $b$ are attributed to the fact that the modified geometry is relatively close to a scaled geometry $(D$ $=W$ ), for which the exact solution is achieved with a single term.

\subsection{Nearly linear dependent basis vectors}

Consider again the fifty-bar truss shown in Fig. $7 a$. Deleting ten diagonal members, the modified design is shown in Fig. 8. Despite the relatively large number of deleted members, the exact solution has been achieved with only three basis vectors. The $\beta$ values obtained for the basis vectors [Eq.(28)], $\cos \beta_{1,2}=0.9621, \cos \beta_{2,3}=1.000$, show that the second and the third basis vectors determined by the CA method are linearly dependent.

\section{CONCLUSIONS}

Some typical cases where exact or accurate solutions can be achieved by the CA method have been presented. It has been shown that exact solutions are obtained in the general case where a basis vector is a linear combination of the previous vectors, and also in cases of low rank modifications to structures or scaling of the initial stiffness matrix. In general the 
CA method does not provide exact solutions, but the solutions presented in the paper explain the high accuracy achieved with a small number of basis vectors. It has been observed that in many cases, where the basis vectors come close to being linearly dependent, accurate solutions have been achieved. Accurate solutions have been achieved also in cases of changes in a small number of elements or when the angle between the two vectors representing the initial design and modified design is small.

The main observations that have been made from the numerical examples are as follows:

a. The reduced basis coefficients can change significantly the convergence properties of the series of basis vectors. Accurate results are obtained by the CA method even in cases where the series of basis vectors diverges.

$b$. The direction of change and the magnitude of changes in the design have a significant effect on the accuracy of the approximations.

c. For any given direction vector $\Delta \mathbf{X}^{*}$, the magnitude of $\alpha$ determines the value of $\theta$. For given number of basis vectors and direction of change $\Delta \mathbf{X}^{*}$, the accuracy of the results depends on $\alpha$. The larger $\alpha$ is, the larger is the angle $\theta$ and more basis vectors will be needed to achieve adequate accuracy.

d. A specific $\theta$ may correspond to many different combinations of $\Delta \mathbf{X}^{*}$ and $\alpha$. Various modified designs obtained by scaling a specific design correspond to the same $\theta$ angle. Similar accuracy has been achieved for the above designs, even in cases where the design changes are very large.

\section{ACKNOWLEDGEMENT}

This work has been partially supported by the US Army Automotive Research Center under the sponsorship of TACOM, and by the General Motors Satellite Research Laboratory at the University of Michigan, Ann Arbor. This support is gratefully acknowledged.

\section{REFERENCES}

1 Kirsch, U., "Efficient reanalysis for topological optimization," Structural optimization, Vol. 6, 1993, pp. 143-150.

2 Kirsch, U. "Improved stiffness-based first-order approximations for structural optimization" AIAA Journal, Vol., 33, 1995, pp. 143-150.

3 Kirsch, U. and Liu S., "Exact structural reanalysis by a first-order reduced basis approach", Structural Optimization, Vol. 10, 1995, 153-158.

$4 \quad$ Kirsch, U., and Liu, S., "Structural Reanalysis for General Layout Modifications", AIAA Journal, Vol. 35, No. 2, 1997, pp. 382-388.

5 Kirsch, U., "Efficient-accurate approximations for structural optimization", AIAA Journal, Vol. 37, No. 12, 1999, pp. 1663-1669.

6 Aktas A and Moses, F., "Reduced basis eigenvalue solutions for damaged structures,"
Mechanics of Structures and Machines, Vol. 26, 1998, pp. 63-79.

7 Leu, L-J. and Huang, C-W., "A reduced basis method for geometric nonlinear analysis of structures", IASS Journal, Vol. 39, No. 127, 1998, pp. 71-75.

8 Kirsch, U., "Combined approximations - a general approach for structural optimization", to be published, Structural Optimization (in press).

9 Sherman, J. and Morrison, W. J., "Adjustment of an inverse matrix corresponding to changes in the elements of a given column or a given row of the original matrix", Ann. Math. Statist., Vol. 20, 1949, p. 621.

10 Woodbury, M., "Inverting modified matrices" Memorandum Report 42, Statistical research group, Princeton University, Princeton, NJ, 1950.

11 Akgun, M. A. Garcelon, J. H. and Haftka, R. T., "Fast exact linear and nonlinear structural reanalysis and the Sherman-MorrisonWoodbury formulas", presented in the NASA/ISSMO First Internet Conference on Approximations and Fast Reanalysis in Engineering Optimization, June 1998.

12 Nelson, S.A. and Papalambros, P.Y., "A modified trust region algorithm for hierarchical nonlinear programming", Structural Optimization, Vol. 16, 1998, pp. 19-28.

13 Kirsch, U. "Rational move limits for approximate structural optimization", $A S C E$ Journal of Structural Engineering, Vol. 123, No. 2, 1997, pp. 210-217. 
Table 1. Displacements for various modified designs (ten-bar truss)

\begin{tabular}{|c|c|c|c|c|}
\hline \multirow{2}{*}{$\begin{array}{l}\text { Method } \\
\text { Number of basis vectors }\end{array}$} & \multicolumn{3}{|c|}{$\mathrm{CA}$} & \multirow[t]{2}{*}{ Exact } \\
\hline & 2 & 3 & 4 & \\
\hline \multirow{8}{*}{$\begin{array}{l}\text { Case } A . \theta=14^{0} \\
\text { (small change) }\end{array}$} & 1.36 & & & 1.37 \\
\hline & 3.59 & & & 3.56 \\
\hline & 1.76 & & & 1.77 \\
\hline & 8.23 & & & 8.25 \\
\hline & -2.06 & & & -2.10 \\
\hline & 8.62 & & & 8.65 \\
\hline & -1.44 & & & -1.45 \\
\hline & 3.92 & & & 3.89 \\
\hline \multirow{8}{*}{$\begin{array}{l}\text { Case } B . \theta=34^{0} \\
\text { (medium change) }\end{array}$} & 0.50 & 0.52 & & 0.52 \\
\hline & 1.53 & 1.46 & & 1.49 \\
\hline & 0.71 & 0.76 & & 0.77 \\
\hline & 3.56 & 3.63 & & 3.65 \\
\hline & -0.89 & -0.98 & & -0.98 \\
\hline & 3.77 & 3.87 & & 3.90 \\
\hline & -0.54 & -0.55 & & -0.55 \\
\hline & 1.71 & 1.64 & & 1.62 \\
\hline \multirow{8}{*}{$\begin{array}{l}\text { Case } C . \quad \theta=41^{0} \\
\text { (large change) }\end{array}$} & 0.28 & 0.29 & 0.29 & 0.30 \\
\hline & 0.90 & 0.84 & 0.88 & 0.90 \\
\hline & 0.41 & 0.45 & 0.47 & $* 0.52$ \\
\hline & 2.10 & 2.17 & 2.19 & $* 2.17$ \\
\hline & -0.53 & -0.61 & -0.62 & -0.60 \\
\hline & 2.24 & 2.34 & 2.37 & 2.40 \\
\hline & -0.30 & -0.31 & -0.31 & -0.30 \\
\hline & 1.01 & 0.95 & 0.93 & 0.90 \\
\hline \multirow{8}{*}{$\begin{array}{l}\text { Case } D . \theta=14^{0} \\
\text { (very large change) }\end{array}$} & 0.14 & & & 0.14 \\
\hline & 0.36 & & & 0.36 \\
\hline & 0.18 & & & 0.18 \\
\hline & 0.82 & & & 0.83 \\
\hline & -0.21 & & & -0.21 \\
\hline & 0.86 & & & 0.87 \\
\hline & -0.14 & & & -0.15 \\
\hline & 0.39 & & & 0.39 \\
\hline
\end{tabular}

* Joint 2 is practically eliminated 
Table 2. Values of $\cos \beta_{i, i+1}$, various modified designs and basis vectors (ten-bar truss)

\begin{tabular}{lllll}
\hline \hline Vectors & 1,2 & 2,3 & 3,4 & 4,5 \\
\hline Cases $A, B, C$ & 0.9989 & 0.9992 & 0.9994 & 0.9997 \\
Case $D$ & 0.9998 & 0.9999 & 0.9999 & 0.9999 \\
\hline
\end{tabular}

Table 3. Exact solutions, various cases of deletion of members (ten-bar truss)

\begin{tabular}{llllllllll}
\hline \hline Deleted & \multicolumn{10}{c}{ Displacements } \\
\cline { 2 - 10 } members & \multicolumn{1}{c}{1} & \multicolumn{10}{c}{2} & 3 & 4 & 5 & 6 & 7 & 8 \\
\hline $2+6$ & 2.40 & 5.80 & $*$ & $*$ & -3.60 & 15.18 & -2.40 & 5.80 \\
$4+9$ & 2.11 & 4.67 & 3.30 & 13.62 & $*$ & 14.81 & -1.35 & 5.57 \\
$5+8+9$ & $*$ & $*$ & 2.40 & 19.76 & -3.60 & 20.96 & -3.60 & 10.38 \\
$4+5+8+9$ & 1.20 & $*$ & 2.40 & 19.76 & $*$ & 20.96 & -3.60 & 10.38 \\
\hline \hline
\end{tabular}

* Irrelevant results

Table 4 First-order approximations, geometrical changes (fifty-bar truss)

\begin{tabular}{|c|c|c|c|c|c|c|}
\hline \multirow[b]{2}{*}{ Joint } & \multirow{2}{*}{\multicolumn{2}{|c|}{ Direction }} & \multicolumn{2}{|c|}{ Case $a$} & \multicolumn{2}{|c|}{ Case $b$} \\
\hline & & & \multirow{2}{*}{$\frac{\text { Exact }}{0.08}$} & \multirow{2}{*}{$\frac{\mathrm{CA} 1}{0.09}$} & \multirow{2}{*}{$\frac{\text { Exact }}{0.20}$} & \multirow{2}{*}{$\frac{\mathrm{CA1}}{0.20}$} \\
\hline & 2 & $X$ & & & & \\
\hline & & $Y$ & 0.08 & 0.11 & 0.24 & 0.25 \\
\hline & 3 & $X$ & 0.15 & 0.16 & 0.38 & 0.38 \\
\hline & & $Y$ & 0.28 & 0.35 & 0.88 & 0.90 \\
\hline & 4 & $X$ & 0.21 & 0.22 & 0.54 & 0.54 \\
\hline & & $Y$ & 0.60 & 0.69 & 1.87 & 1.90 \\
\hline & 5 & $X$ & 0.27 & 0.26 & 0.67 & 0.68 \\
\hline & & $\mathrm{Y}$ & 1.01 & 1.12 & 3.18 & 3.21 \\
\hline & 6 & $X$ & 0.31 & 0.29 & 0.79 & 0.79 \\
\hline & & $\mathrm{Y}$ & 1.51 & 1.60 & 4.74 & 4.79 \\
\hline & 7 & $\mathrm{X}$ & 0.35 & 0.32 & 0.88 & 0.88 \\
\hline & & $Y$ & 2.07 & 2.13 & 6.54 & 6.58 \\
\hline & 8 & $\mathrm{X}$ & 0.38 & 0.34 & 0.96 & 0.95 \\
\hline & & $\mathrm{Y}$ & 2.69 & 2.69 & 8.50 & 8.54 \\
\hline & 9 & $X$ & 0.40 & 0.35 & 1.01 & 1.01 \\
\hline & & $Y$ & 3.36 & 3.27 & 10.60 & 10.63 \\
\hline & 10 & $X$ & 0.41 & 0.35 & 1.04 & 1.04 \\
\hline & & $Y$ & 4.05 & 3.86 & 12.79 & 12.81 \\
\hline & 11 & $X$ & 0.42 & 0.35 & 1.05 & 1.05 \\
\hline & & $\mathrm{Y}$ & 4.75 & 4.45 & 15.02 & 15.02 \\
\hline
\end{tabular}




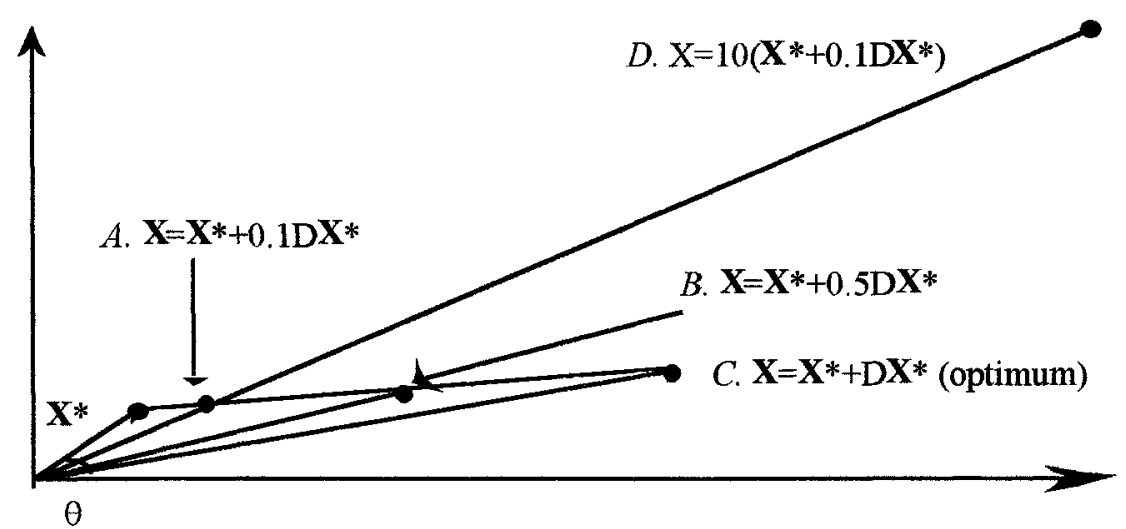

Fig. 1. Various modified designs shown in the space of $\mathbf{X}^{*}$ and $\Delta \mathbf{X}^{*}$

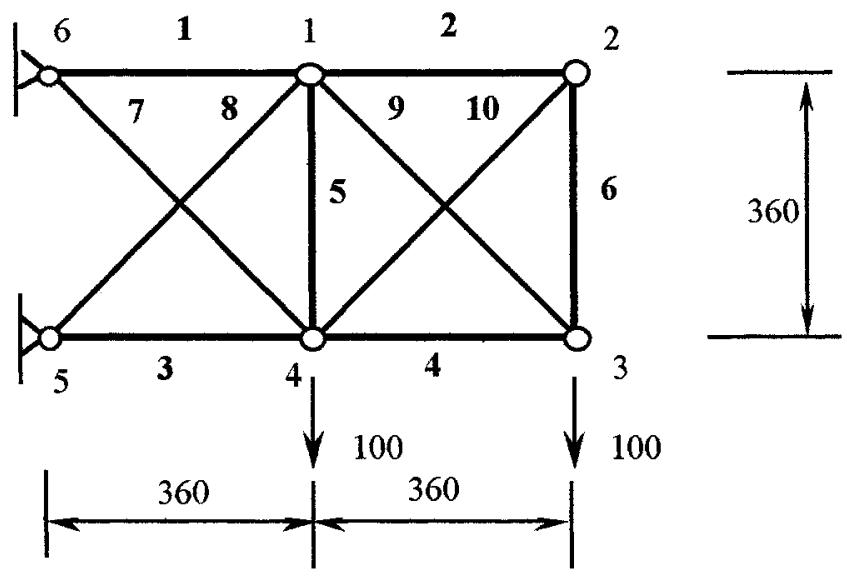

Fig. 2. Ten-bar truss.

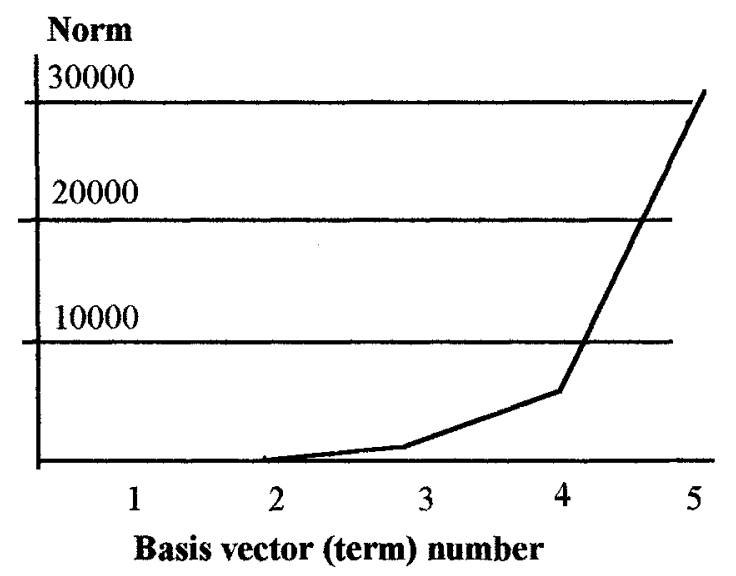

Fig. 3. Norm of the original basis vectors $(\alpha=1.0)$. 


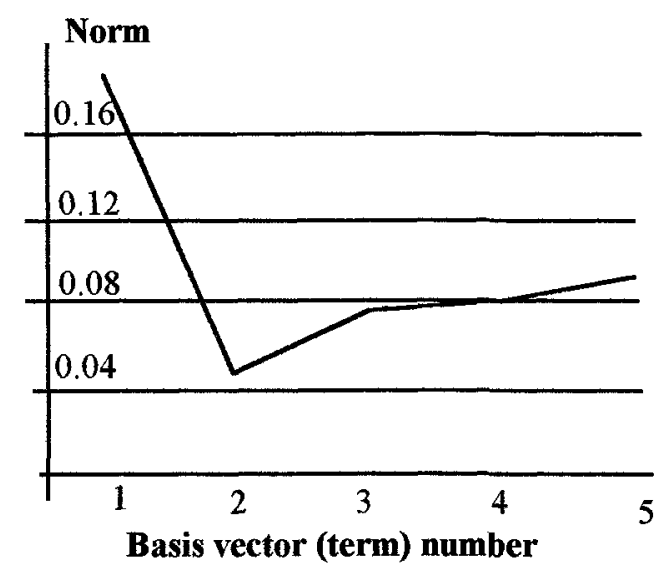

Fig. 4. Norm of the uncoupled vectors.

(a)

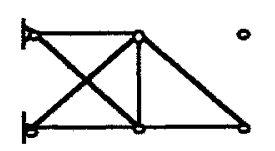

(b)

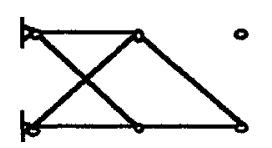

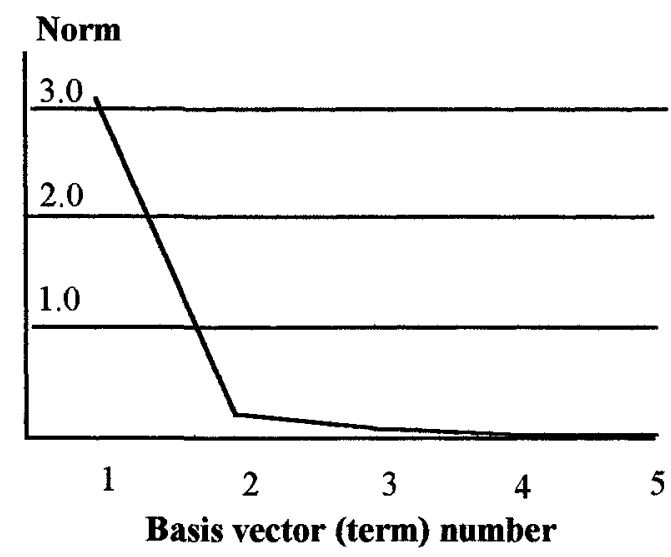

Fig. 5. Norm of the CA terms.

Fig. 6. Ten-bar truss, modified topologies.
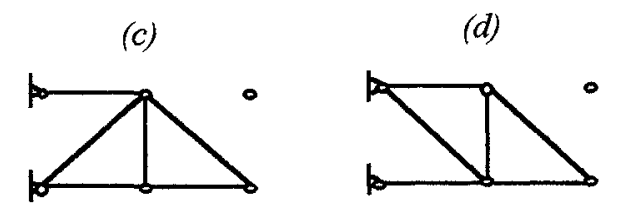

(a)

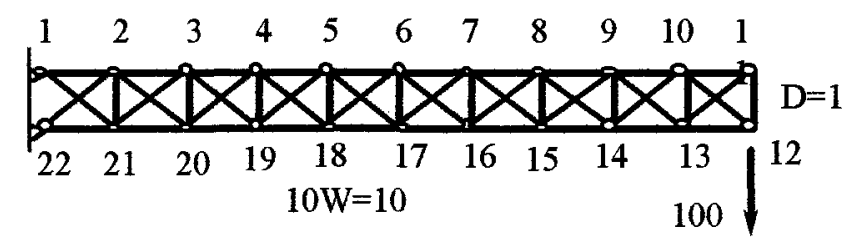

(b)

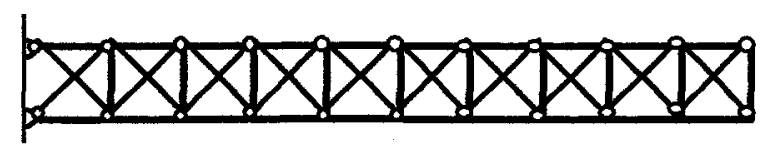

(c)

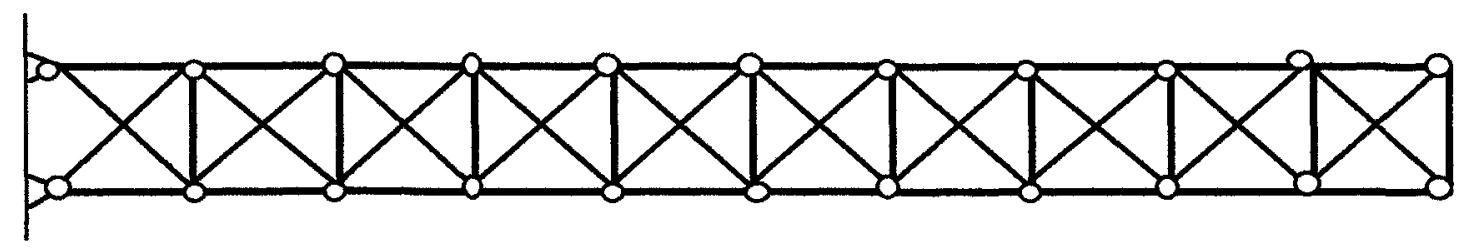

Fig. 7. Fifty-bar truss, initial and modified geometries.

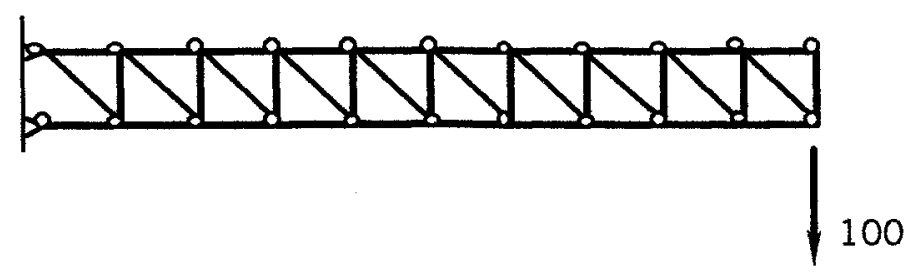

Fig. 8. Fifty-bar truss, modified topology. 